\title{
Herbert Gleiter to Receive 2008 Von Hippel Award
}

The 2008 Von Hippel Award, the Materials Research Society's highest honor, will be presented to Herbert Gleiter, Forschungszentrum Karlsruhe, Institute for Nanotechnology, Karlsruhe, Germany. Gleiter is being recognized "for his imaginative experiments on the role of defects that have led to new insights into the importance of length scale in materials and have resulted in many new applications." Gleiter will accept the honor during the awards ceremony at the 2008 MRS Fall Meeting in Boston on Dec. 3 at 6:00 p.m. in the Grand Ballroom of Sheraton Boston Hotel, where he will then present his award lecture.

Throughout his career, Gleiter has been, and continues to be, an outstanding researcher in the field of materials science. His new and visionary concepts have laid the foundation for new developments in materials science, and also impacted related areas of research, such as physics and chemistry. He has made major contributions in the areas of nanocrystalline materials, the structure and properties of inter-crystalline interfaces, high-strength materials, and materials with electronically tunable properties.

Gleiter was among the first to recognize the potential of nanocrystalline materials, which is one of the fastest growing technology areas in the marketplace. Several years prior to the first experimental evidence of the new structure of nanocrystalline materials, Gleiter predicted their main structural details and the potential for novel properties. The original concept of nanocrystalline materials was based on the idea that a new class of solids can be produced by a reduction of the grain size to the dimensions of a few interatomic distances. Because the atomic structure at these interfaces is different from that in perfect crystals, nanocrystalline materials have properties which differ from those of crystalline and amorphous materials, in some cases by orders of magnitude

Gleiter's pioneering influence in the study of the role of defects in intercrystalline interfaces resulted in two important discoveries: the proof of the existence of grain boundary dislocations, and the development (in collaboration with B. Chalmers and G. Bishop) of the structural unit model, which still remains the basis of all grain boundary structure models.

The development of high-strength materials by precipitation hardening has

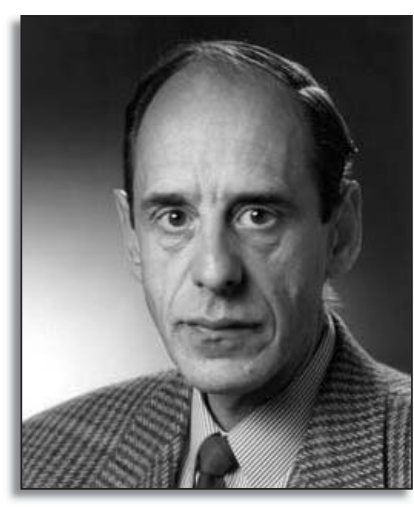

Herbert Gleiter

numerous applications in industry. Gleiter discovered the basic hardening mechanism responsible for the strength of high-temperature alloys with ordered precipitations. Using transmission electron spectroscopy, he also identified the dislocation mechanisms of the prismatic cross slip, which play an important role in the high-temperature deformation of precipitation hardened alloys.

The realization that properties of a material might be "tuned" by making the microstructural length scale comparable to the electronic screening length led Gleiter to the discovery of a new class of materials. Using this concept, he demonstrated that the surface stresses in nanoporous materials exposed to an electrolyte can be reversibly changed by the application of a control potential, leading to reversible length changes. Similarly, electrical conductivity, ferromagnetic, and optical properties can be tuned reversibly.

Gleiter has also made important contributions in the areas of ferroelectric materials, polymer physics, and phase transitions. An important result was the development of a method to prevent the melting of crystals at their normal melting point, allowing the examination of overheated crystals, which had not previously been possible.

Gleiter was awarded a PhD degree in physics from the University of Stuttgart, Germany, in 1966 and a DSc degree in materials science from the University of Bochum, Germany, in 1970. He was an assistant professor at the University of Göttingen in Germany, from 1966 to 1967, before accepting a research fellowship at Harvard (1967-1970) and an assistant professorship at the Massachusetts Institute of Technology (1971). In 1972, he returned to
Germany as Professor of Materials Science at the University of Bochum, and in 1983, he was appointed Chair Professor of Materials Science at University of Saarland in Saarbrücken. In 1987, Gleiter became the Founding Director of the Institute for New Materials in Saarbrücken. From 1994 to 1998, he was a member of the Executive Board of Forschungszentrum Karlsruhe, and was Director of the newly founded Institute of Nanotechnology at Forschungszentrum Karlsruhe from 1998 to 2004. Throughout his career, Gleiter has received numerous professional awards. In 1972, he received the Masing Prize of the German Society of Metals and in 1984, he was awarded the Alcoa Prize from the Alcoa Foundation, Pittsburgh. In 1988, he was awarded the Leibniz Prize of the German National Science Foundation, which carried a three million DM (\$US 2.5 million) research grant. The Humboldt Foundation and the Max Planck Society in Germany awarded Gleiter the Max-Planck Prize in 1993. He received the Gold Medal of the Federation of European Materials Research in 1995, followed by the Heyn Medal of the German Society for Materials in 1998, and the Alexander-von-Humboldt Foundation awarded Gleiter the Heisenberg Medal in 2000, and the Humboldt Medal in 2006. Gleiter has been a member of the German Academy of Science, Leopoldina, since 2004, and he is a member of the U.S. National Academy of Engineering, Foreign Honorary Member of the American Academy of Arts and Sciences, and a member of the Indian National Academy of Engineering.

Gleiter's contributions to the international scientific literature in various fields of materials science have been prolific, and he has published 150 papers since the establishment of the ISI system in 1988. One of his most important papers received 1469 citations, followed by nine publications with more than 100 citations.

The MRS Von Hippel Award includes a $\$ 10,000$ cash prize, honorary membership in MRS, and a unique trophy-a mounted ruby laser crystal, symbolizing the many-faceted nature of materials research. The award recognizes those qualities most prized by materials scientists and engineers-brilliance and originality of intellect, combined with vision that transcends the boundaries of conventional scientific disciplines, as exemplified by the life of Arthur von Hippel (http://vonhippel.mrs.org). 